\title{
Diurnal seismic periodicity corroborates a mechanism involving solar and lunar gravitational pulls and gravity of the Earth
}

Kaiqiao Yang ${ }^{1}$, Qiuyun Liu ${ }^{1, *}$

${ }^{1}$ School of Life Sciences, Sun Yat-sen University, Guangzhou 510275, China.

${ }^{*}$ Corresponding author: Qiuyun Liu, Ph.D, School of Life Sciences, Sun Yat-sen University, Guangzhou 510275, China. Tel: [+86 (020) 84110296]; E-mail: 1sslqy@mail.sysu.edu.cn 


\begin{abstract}
:
The diurnal seismic frequency peaks at 0:00, 12:00, 18:00 local mean time can be explained by the trio interactions of solar and lunar gravitational pulls and the gravity of the Earth impacting where potential energy is present. The revolution of the Earth also contributes to the stress in the crust, which is perpendicular to the stress generated along the direction of the Earth's self-rotation.
\end{abstract}

\title{
Keywords:
}

diurnal seismic periodicity; solar and lunar gravitational pulls; gravity of the Earth; potential energy 
Earthquakes are likely to be triggered by the interplay of solar and lunar gravitational pulls and the gravity of the Earth, which gives rise to the exact periodicity of earthquakes with similar magnitudes occurring in the same area. ${ }^{1-8}$. Recently, fortnightly and low-frequency earthquake patterns have been identified from millions of earthquakes near the San Andreas fault ${ }^{9}$. It is also found that very large earthquakes often occur when maximum amplitude of tidal stress appears ${ }^{10}$, but this trend is not obvious for small earthquakes. Fortnightly and diurnal seismic periodicities have been found in the western regions of the United States ${ }^{11}$. For simplicity here we consider solar pull only. At 0:00 midnight, the crust begins to accelerate, and small mass in the west accelerates more quickly, crashing into large mass in the adjcent east which accounts for the seismic frequency peak at 1 to $2 \mathrm{am}^{12}$. At 12:00, large mass decelerates more slowly, crashing into the adjcent small mass in the east, which explains the peak at this time ${ }^{12}$. After 18:00 local mean time, solar pull drives Earth crust backward and downward, while the horizontal component of solar pull gradually loses strength. At this time, the small mass in the east side impacts the large mass with larger inertia in the west side. The revolution of the Earth is also responsible for the stress generated in the crust, which is perpendicular to the stress generated in the direction of the Earth's self-rotation ${ }^{13-14}$.

\section{Conflict of interest statement}

The authors declare no conflict of interests.

\section{Acknowledgements}

Q.L. was supported by grants from the Science and Technology Transformation Program of Sun Yat-sen University of China (33000-18843234), Guangzhou Science and Technology Program (201804010328) and Guangdong Science and Technology Program (2016B020204001). We are grateful to Yan Shi for editing.

\section{References}

1. He R, He S, Nuertai X, et al. The faster acceleration of smaller masses in the west of Americas. Science 2017; http://science.sciencemag.org/content/354/6315/1027/tab-e-letters.

2. Yan S, Wang X, Qi J, Liu Q. Volcanoes or earthquakes: Wrist wrestling. Science, 2019; http://science.sciencemag.org/content/358/6370/1520/tab-e-letters.

3. Yan $\mathrm{S}, \mathrm{Xu} \mathrm{X}$, Chen L, et al. Earthquakes: The rocking cradle. Science 2019; https://science.sciencemag.org/content/354/6315/1027/tab-e-letters.

4. Yan S, Li J, Liu Q. Earthquakes-an intricate trio dance of gravitation. Science 2018; http://science.sciencemag.org/content/354/6315/1027/tab-e-letters.

5. $\mathrm{Xu} \mathrm{H,} \mathrm{Wu} \mathrm{J,} \mathrm{Liu} \mathrm{Q.} \mathrm{Earth's} \mathrm{self-rotation:} \mathrm{high-velocity} \mathrm{movements} \mathrm{manifested} \mathrm{in}$ the solar and lunar gravitational fields. Science 2019; https://science.sciencemag.org/content/354/6308/88/tab-e-letters.

6. Yang $\mathrm{K}, \mathrm{Xu} \mathrm{H}$, Liu W, Liu Q. Why several megaquakes strike around noon? Science 2019; https://science.sciencemag.org/content/354/6308/88/tab-e-letters. 
7. Yang K, Liu J, Liu Q. The basis for continually-distributing daily seismic activities. Science 2019; https://science.sciencemag.org/content/354/6308/88/tab-e-letters).

8. Yan $\mathrm{S}, \mathrm{Du}, \mathrm{Y}, \mathrm{Xu} \mathrm{H}$, et al. Filtering out non-specific animal behavior to better predict impending earthquakes. OSF Preprints. 2020; DOI: 10.31219/osf.io/bs3qd . https://doi.org/10.31219/osf.io/bs3qd

9. Van der Elst NJ, Delorey AA and Shelly DR. Fortnightly modulation of San Andreas tremor and low-frequency earthquakes. Proc Natl Acad Sci USA 2016; 113: 8601-5.

10. Ide S, Yabe $\mathrm{S}$ and Tanaka Y. Earthquake potential revealed by tidal influence on earthquake size-frequency statistics. Nat Geosci 2016; 9: 834-7.

11. Atef $\mathrm{AH}, \mathrm{Liu} \mathrm{KH}$ and Gao SS. Apparent weekly and daily earthquake periodicities in the Western United States. Bull Seismol Soc Am 2009; 99: 22739.

12. Hao J, Zhang J, Yao Z. Evidence for diurnal periodicity of earthquakes from midnight to daybreak, National Science Review 2019; 6(5): 1016-1023.

13. Lu X, Ran H, Zheng R, et al. The revolution of the Earth around the sun causes the vortex-induced vibration of south-north trending bridges and impacts seismic activities. OSF Preprints. 2020; DOI: 10.31219/osf.io/ycjhz. https://doi.org/10.31219/osf.io/ycjhz

14. Zheng R, Jin W, Zeng T, et al. The Uplift of the Qinghai-Tibet Plateau: The Driver Behind the Scene. OSF Preprints 2020. DOI: 10.31219/osf.io/hpvk7. https://osf.io/hpvk7 